\title{
Correspondence
}

\section{The doctor and seclusion}

Sir: I was saddened by the innocence and ignorance shown by Drs Okhai and McLaren in claiming that the supervision of seclusion of patients should be no part of a trainee psychiatrist's job (Psychiatric Bulletin. April 1997, 21, 227). Are they not concerned with the general well-being of their patients as well as with specific mental illnesses? If one of their patients suddenly appeared with two black eyes would they not think that they ought at least to ask why? Are they really so unaware of all the abuses, physical, emotional and sexual, sometimes suffered by vulnerable patients, children and old people, in homes and hostels as well as in psychiatric and even general wards? The newspapers today sometimes tell us about such matters, and the history of psychiatry in the 19th and 20th century is full of scandals, public enquiries and regulations and laws passed to try to prevent their recurrence.

Seclusion (equals imprisonment without trial) should not be simply a way of doing without staff, or of satisfying a private grudge; it must be openly justified; it must only be for a limited prescribed period; it must not be easily repeated; and there must be frequent, regular checks on the secluded person's wellbeing in the cell. It will not always be carried out properly unless it is inspected and written down in the case record for evidence. External inspection is essential, because nurses are loyal to their colleagues and will not report against them, doctors too are loyal to their fellows, while administrators want things hushed up. Often no-one knows quite how to deal with abusers if they are discovered.

Physical and mental abuse seems to be a disease of institutions rather than the acts of one or two individuals unsuited to their work (paedophile in a children's ward, sadistic psychiatric nurse, etc.,). It tends to be a reflection of poor staff morale; overwork and long hours with low pay; an administration ignoring complaints and offering no support, particularly with overcrowding; lack of job satisfaction; and patients who are sectioned, irritating, perverse or obstructive. Any of us is a potential abuser if the environment is bad enough.

The trainee needs to be involved in seclusion, not because it is therapy (which it is not) but to prevent it becoming anti-therapy, and to use the opportunity to get to know (and support) nursing colleagues. A team pulls together, it is not a set of individuals each doing their own thing.

JOHN CRAMMER, South Grange, Steeple Aston, OX6 3SS

Authors' reply: John Crammer's letter suggests he has missed the point of our paper. His letter belies an ambivalent attitude towards psychiatric nurses, as on the one hand he recommends that trainees should 'get to know and support nursing colleagues' while on the other he views them as potential 'abusers' likely to cover for each other's malpractice. The physical abuse of patients has been, and can only be, addressed by increasing the professionalism of psychiatric nursing. Comments such as those made by $\mathrm{Dr}$ Crammer undermine this process. Seclusion is a nursing intervention and responsibility for documentating and monitoring its use should reside within that profession.

If monitoring by one professional group of another's practice is required, and our view is that it is not, is it appropriate to ask junior psychiatrists, who may be in their first psychiatric post, to oversee the actions of experienced nursing staff? If Dr Crammer is serious in his concerns should he not be advocating that consultants be responsible for 'external inspection'?

We agree with Dr Crammer that the therapeutic effects of seclusion are uncertain. Psychiatrists should be working with nursing colleagues, through clinical audit, to minimise its use. We are still of the view that the current involvement of trainee psychiatrists is inappropriate and needs to be reconsidered.

M. F. OKHAI, Registrar in Psychiatry, St Martin's Hospital, Littlebourne Road, Canterbury CT1 1AZ; and P. M. MCLAREN, Senior Lecturer in Psychiatry. UMDS Division of Psychiatry and Psychology, Guy's Hospital SE1 9RT

\section{National survey of psychiatric intensive care units}

Sir: Beer et al (Psychiatric Bulletin, March 1997. 21, 142-144) report the results of a postal survey sent to pharmacists who were asked to comment upon the characteristics of their local psychiatric intensive care units (PICUs). The questions asked of these pharmacists are not included in 